This is an electronic reprint of the original article. This reprint may differ from the original in pagination and typographic detail.

Author(s): Koponen, Mikko; Hohenester, U.; Hakala, Tommi; Toppari, Jussi

Title: $\quad$ Absence of mutual polariton scatterings for strongly coupled surface plasmon polaritons and dye molecules with large Stokes shift

Year: $\quad 2013$

Version:

Please cite the original version:

Koponen, M., Hohenester, U., Hakala, T., \& Toppari, J. (2013). Absence of mutual polariton scatterings for strongly coupled surface plasmon polaritons and dye molecules with large Stokes shift. Physical Review B, 88, Article 085425.

https://doi.org/10.1103/PhysRevB.88.085425

All material supplied via JYX is protected by copyright and other intellectual property rights, and duplication or sale of all or part of any of the repository collections is not permitted, except that material may be duplicated by you for your research use or educational purposes in electronic or print form. You must obtain permission for any other use. Electronic or print copies may not be offered, whether for sale or otherwise to anyone who is not an authorised user. 


\title{
Absence of mutual polariton scattering for strongly coupled surface plasmon polaritons and dye molecules with a large Stokes shift
}

\author{
Mikko A. Koponen, ${ }^{1}$ Ulrich Hohenester, ${ }^{2}$ Tommi K. Hakala, ${ }^{3}$ and J. Jussi Toppari ${ }^{1, *}$ \\ ${ }^{1}$ Nanoscience Center, Department of Physics, P.O. Box 35, FI-40014 University of Jyväskylä, Finland \\ ${ }^{2}$ Institut für Physik, Karl-Franzens-Universität Graz, Universitätsplatz 5, 8010 Graz, Austria \\ ${ }^{3}$ Department of Applied Physics, Aalto University School of Science, P.O. Box 15100 FI-00076 AALTO, Finland
}

(Received 21 December 2012; revised manuscript received 25 June 2013; published 21 August 2013)

\begin{abstract}
The understanding and control of the dynamics of hybrid modes consisting of strongly coupled surface plasmon polaritons and molecular excitations of dye molecules is of great timely interest, as it allows one to tailor interactions between optical signals as needed for active all-optical devices. Here we utilize dye molecules with an especially large Stokes shift to demonstrate the absence of mutual scatterings among the strongly coupled hybrid modes. We employ a quantum mechanical three-level model and show that the hybrid modes decay via dephasing and internal relaxation of the molecules to a fluorescing state of the dye, which can be used as a measure for the decay. Our results provide essential information about the dynamics of the strongly coupled modes.
\end{abstract}

DOI: 10.1103/PhysRevB.88.085425

PACS number(s): 78.68. $+\mathrm{m}, 34.35 .+\mathrm{a}, 42.50 . \mathrm{Ct}$

\section{INTRODUCTION}

Surface plasmon polaritons (SPPs) have attracted considerable interest in recent years because of numerous foreseen applications. ${ }^{1-3}$ The evanescent nature and the extremely confined mode volumes of these coupled excitations between electromagnetic fields and free electrons of the metal strongly enhance light-matter interaction at the nanoscale ${ }^{4-9}$ and open routes towards photonics beyond the diffraction limit. ${ }^{3,10-15}$ Strong coupling between SPPs and optically active molecular excitations (MEs) manifests itself through the formation of new hybrid states, ${ }^{16}$ i.e., polaritons between MEs and SPPs (not to be confused with pure SPP), which exhibit Rabi splittings up to several hundreds of $\mathrm{meV}$ depending on the molecular species and concentration. ${ }^{17-25}$ However, the inhomogeneous broadening, practically always present in the assemblies of organic molecules, decreases the observed Rabi splitting if the width of the broadening is close to the coupling strength - and also gives rise to a coexistence of noncoupled MEs and the polaritons. ${ }^{26}$ This results in a pure ME fluorescence appearing together with the luminescence of polaritons in a strongly coupled system ${ }^{18-20}$ (with similar results for the cavity photon polaritons ${ }^{27-29}$ ).

Earlier studies of strongly coupled SPP-ME systems have reported that the luminescence of polaritons with an energy higher than the ME, the so-called upper polariton branch (UPB), is usually very weak despite the large incoupled intensity monitored via reflectance ${ }^{18-21}$ (standard Kretschmann measurement, ${ }^{30}$ see Fig. 1). Despite these clear and consistent observations, the relaxation dynamics of the polaritons into the noncoupled MEs, as well as scatterings among them, are still not fully understood. ${ }^{25,31,32}$ Recent time-resolved studies have shed some light on the issue, ${ }^{33,34}$ and have demonstrated Rabi oscillations as a clear signature of strong coupling. ${ }^{35}$ However, at the same time there has been significant evidence about unexpectedly long lifetimes of the polaritons, ${ }^{36}$ suggesting more pronounced scattering or maybe even thermalization among them.

Since the molecular fluorescence is directly related to the ME occupation, it provides an ideal tool for studying the polariton dynamics. So far all experiments have been carried out with molecules whose fluorescence energy coincides with the energy of the polariton modes lower than the ME, the lower polariton branch (LPB), ${ }^{19,20,22}$ or with molecules/aggregates without any Stokes shift, ${ }^{18,21,24,25}$ which makes the ME fluorescence undistinguishable in luminescence measurements or makes the recoupling of the ME fluorescence back to LPBs very efficient. ${ }^{37,38}$ To overcome this problem, in this paper we employ a fluorophore 9-diethylamino-benzo[a]phenoxazin-5one, commonly referred to as Nile Red (NR), which has a very high Stokes shift $\geqslant 350 \mathrm{meV}$, also when embedded in polymers. ${ }^{39}$ This shift is about three times higher than for previously studied dyes, such as Rhodamine $6 \mathrm{G}(\sim 112 \mathrm{meV}) .{ }^{19}$ Thus, the fluorescence can be separated from the luminescence of polaritons and SPPs, and the recoupling to LPB is weaker. In addition, polarization can be used for an even better separation of the signals, as the ME fluorescence is mostly unpolarized in contrast to the polarized luminescence of the polaritons or uncoupled SPPs. With this setup, we show that the hybrid SPP-ME polaritons decay to MEs via dephasing and internal relaxation of the molecules. Our results provide essential information on the dynamics of the strongly coupled modes.

\section{METHODS}

\section{A. Sample fabrication}

The samples used in this study consist of a thin layer of silver on a glass substrate with a polymer matrix on top with the NR embedded in. The fabrication started with a thorough cleaning of a microscope glass slide with a thickness of $1.5 \mathrm{~mm}$ and refractive index of 1.52. The silver layer was formed on top of the glass by electron-beam evaporation in ultrahigh vacuum $\left(10^{-8} \mathrm{mbar}\right)$. The evaporation rate of $2-4 \mathrm{~nm} / \mathrm{s}$ was chosen to obtain smooth surfaces. The thickness of the metal layer is $\sim 50 \mathrm{~nm}$ and the surface roughness $\sim 2.1 \mathrm{~nm}_{\mathrm{rms}}$, which were verified with atomic force microscopy (AFM) (Veeco Dimension 3100).

SU-8 epoxy-based polymer negative resist (Microchem SU-8 2025) was used as a matrix for the molecules. A suitable 


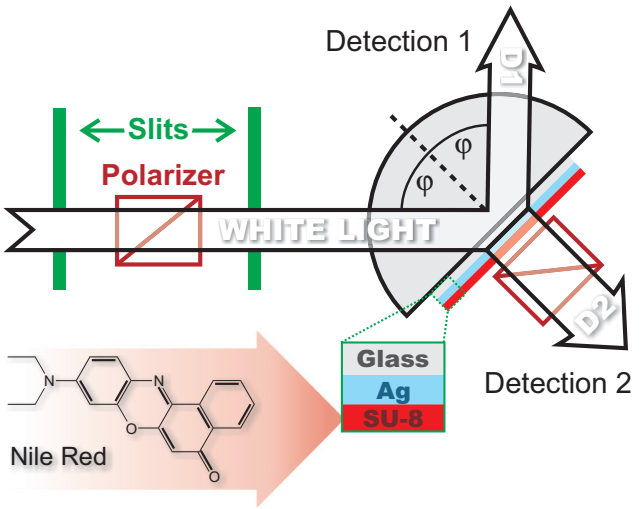

FIG. 1. (Color online) Schematics of the measurement setup and the samples. The sample is mounted on the flat surface of the rotatable hemicylindrical prism by index matching oil. The excitation of SPPs is done by linearly polarized and collimated white light and both the reflected signal (detection 1) and the luminescence signals (detection 2) were collected to a fiber guiding the radiation to a spectrometer. $\varphi$ is the angle of incidence as well as the detection angle in detection 1. The lower left part represents the structure of the sample consisting of two layers, silver and SU-8 polymer with the Nile Red dye embedded, at the top of a glass substrate. Thickness of the silver and polymer layers were about $50 \mathrm{~nm}$.

amount of Nile Red (Sigma-Aldrich) was first dissolved in cyclopentanone and subsequently mixed with SU-8 resist already diluted with cyclopentanone by a 1:6 volume ratio. Further filtering was used to remove any possible aggregates or residues of size above $0.2 \mu \mathrm{m}$. After the filtration the resist was spin-coated on top of the silver with a rate of $6000 \mathrm{rpm}$ resulting in a $\sim 50 \mathrm{~nm}$ thick layer. The obtained layer was used as is without any subsequent baking. For the absorption and fluorescence measurements, reference samples without silver were similarly fabricated.

\section{B. Measurement setup}

For measurements the samples were installed by index matching oil on the flat face of a hemicylindrical prism (ThorLabs) made of BK7 glass with an index of refraction of 1.52. An Oriel 66182 white light source was used for excitation of the SPPs on the silver layer via Kretchmann configuration, ${ }^{30}$ as shown in Fig. 1. The linearly polarized collimated light beam was formed and aligned by two slits having a large separation compared to the beam width, and polarized by a prism polarizer positioned in between the slits (see Fig. 1). The maximum intensity of the excitation used was $\sim 15 \mathrm{~W} / \mathrm{m}^{2}$, which was shown not to induce any nonlinearities on the experiments. The angle of incidence for the incoming light was adjusted manually by rotating the goniometric prism mount. The obtained angular precision was about $0.5^{\circ}$.

The reflected signal (D1) and the luminescent light (D2), i.e., out-scattered polaritons and SPPs as well as fluorescence of the MEs, were collected by a collimating optics assembly (ThorLabs F220SMA-A) connected to an optic fiber guiding the radiation to a Jobin Yvon iHR320 spectrometer equipped with a Jobin Yvon Symphony CCD camera. It should be noted that D1 and D2 signals were not collected simultaneously. An extra polarizer was utilized in front of the D2 collection for improved separation of the luminescence due to out-scattered polaritons and ME fluorescence. The polarizer is positioned before the fiber, between the flat face of the prism and the collection assembly (see Fig. 1), and thus after all the relaxation and scattering processes on the sample.

The absorption of the polymer layers on three samples with different NR concentrations, which we denote as samples NR1, NR2, and NR3 for the NR/SU- 8 mass ratios of $\sim 0.2, \sim 0.45$, and $\sim 1$, respectively, were measured separately, i.e., without the silver layer, by a spectrometer (Perkin Elmer UV/VIS Lambda 850). We checked by separate fluorescence measurements of plain NR/SU-8 films that the NR fluorescence is fully unpolarized, i.e., the recorded transverse electric (TE) and transverse magnetic (TM) spectra coincide, in contrast to the luminescence of the polaritons which is purely TM polarized.

\section{Modeling of the dielectric layer}

The absorption of the NR-polymer film consists of two overlapping maxima and needs to be fitted with two peaks to yield a reliable fit. The measured absorptions from the samples NR1, NR2, and NR3 were fitted with two Gaussian peaks having center wavelengths of 570 and $520 \mathrm{~nm}$, and standard deviations of 148 and $125 \mathrm{~nm}$, respectively. The weaker maximum at lower energies could be due to the formation of J-aggregates, although Nile Red can have several maxima due to its internal structure alone. ${ }^{39}$ The absorption spectra are presented together with the fits in Fig. 2(a).

\section{Calculations by the transfer matrix method}

We use a transfer matrix approach to compute the electromagnetic fields in the layered system, as well as the reflection and transmission coefficients. The model system used is a multilayered structure that consists of semi-infinite dispersionless glass (refractive index 1.52), $50 \mathrm{~nm}$ silver layer with dielectric function calculated using the data from Ref. 40, $50 \mathrm{~nm}$ of resist containing Nile Red, and a semi-infinite air layer. The imaginary part of the dielectric function $\epsilon(\omega)$ of the molecule layer was obtained from the above-mentioned fitting of the two Gaussian peaks to the NR absorption. The real part was calculated from the Kramers-Kronig relation and using a constant of 2.5 for the permittivity of the SU- 8 medium hosting the molecules.

Figure 2(b) shows a compilation of the reflectance spectra calculated by the transfer matrix method, together with the measured ones. The agreement between measurements and the calculations is very good throughout. However, as the NR concentration increases the calculation starts to overestimate the strength of the UPB. This could indicate that the relaxation from the UPB is so fast that it can be detected even through the reflected light. This would further imply that the decoherence time decreases with increasing NR concentration.

For the calculation of the molecular excitation probability we employ the Poynting vector at the interface between the silver film and the NR-doped polymer layer, which describes the transfer of energy into the molecular film. Since for the Kretschmann geometry no light can propagate on the air side, the energy transported into the molecular layer must be absorbed by the molecules, whose subsequent decay can be monitored in fluorescence. 

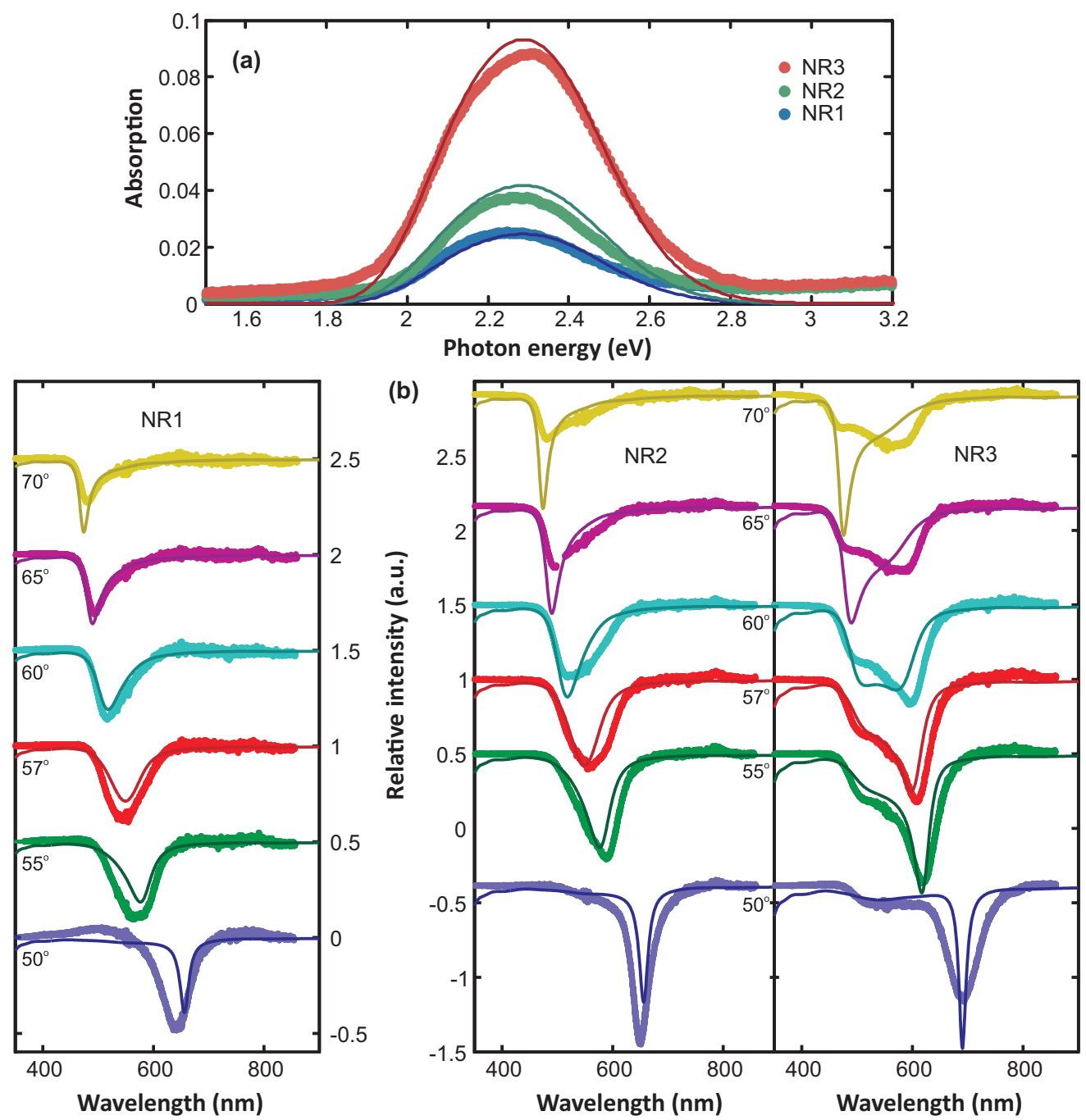

FIG. 2. (Color online) Absorptions and reflectances. (a) Measured absorptions (in the scale from 0 to 1) from the samples NR1, NR2, and NR3 shown as blue, green, and red circles, respectively, together with the double Gaussian fits (solid lines). (b) Measured reflectance (D1) spectra for all the samples and for several angles (solid circles), together with the results obtained from the transfer matrix method (solid lines). The spectra with different angles are shifted vertically for clarity.

\section{E. Coupled oscillator model}

To calculate the molecular fluorescence probability/intensity for each angle of excitation, the dispersion curves obtained from the D1 Kretchmann measurements were fitted with the model of coupled oscillators or strong coupling between MEs and SPPs. The coupled oscillator model yields three polariton branches, i.e., upper (UPB), middle (MPB), and lower (LPB) polariton branches, separated by $\mathrm{ME}_{1} \approx 2.38 \mathrm{eV}$ $(520 \mathrm{~nm})$ and $\mathrm{ME}_{2} \approx 2.17 \mathrm{eV}(570 \mathrm{~nm})$ in energy. These states are then utilized to model mutual scatterings between the polaritons and MEs, as explained later in the results section. Full details of this three-level model can be found in the Appendix.

\section{RESULTS}

\section{A. Measurements of the dispersion curves}

In white-light excitation and for a given angle of excitation $\varphi$, all modes crossing the angle dependent light line are launched [see Fig. 6(a)]. The energies of these modes were obtained from the dips on the measured reflectance spectra D1, shown for several angles in Fig. 2(b). In the case of an assembly of organic molecules, the Rayleigh criterion for the existence of the hybrid polariton mode is relaxed since the linewidth of a molecular absorption is not due to the lifetime broadening but rather due to a large manifold of vibrational sublevels of the molecule. As stated above, the inhomogeneous broadening can also decrease the observed Rabi splitting from the real coupling strength if the width of the broadening is close to the coupling strength, as in our case. ${ }^{26,41}$ Thus, although the widths of the absorption maxima of NR are slightly higher than the observed Rabi splittings, the polariton modes were indeed formed, which could be easily verified and determined by fitting of two or three Gaussian dips. The Rayleigh criterion for the mode visibility still naturally exists, and special care had to be taken to use only a minimum number of dips to produce a valid fit, i.e., to not introduce spurious, additional modes. 


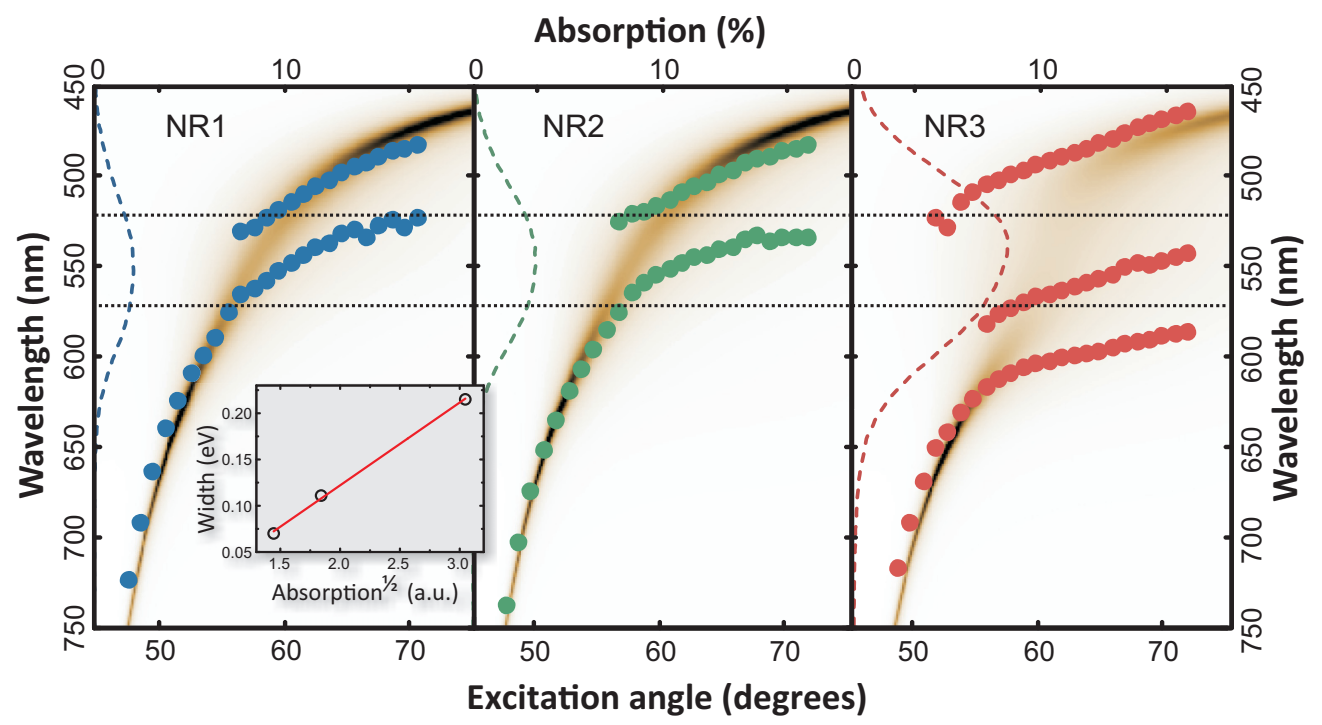

FIG. 3. (Color online) Center wavelengths of the measured dips (circles) in the reflectivity (D1) as a function of the excitation angle, showing clear Rabi splittings in the dispersion curves for all three samples NR1, NR2, and N3 (blue, green, and red, respectively). Overlayed color/grayscale is the reflectance coefficient calculated by the transfer matrix method using the parameters fitted from the absorptions, shown on the left axis with scale on the top axis (dashed lines). Dotted horizontal lines show the center wavelengths of the molecular excitations. The inset shows the widths of the higher $\left(\mathrm{ME}_{1}\right)$ splitting (circles) as a function of square root of the total absorption. The solid line is a linear fit to the points.

Figure 3 presents the center wavelengths of the measured modes/dips for all three samples as a function of the excitation angle, together with the reflectance coefficients calculated by the transfer matrix method using the parameters fitted from the absorptions, which are also shown on the left axis. The measured data are in good agreement with the calculations.

As seen from the figure, a clear Rabi splitting is observed at the higher energy excitation of $\mathrm{NR}, \mathrm{ME}_{1}$, for the samples NR1 and NR2. Similarly, a double Rabi splitting, i.e., splitting at both NR excitations $E_{1} \approx 2.38 \mathrm{eV}$ and $E_{2} \approx 2.17 \mathrm{eV}$, is visible for the sample NR3 with the highest concentration, showing clearly all three polariton branches. The $\mathrm{ME}_{2}$ splitting in the lower concentration samples NR1 and NR2 could not be determined due to narrow splitting and thus too high overlap of the modes. The inset shows the widths of the higher energy splitting as a function of square root of the total absorption, and reveals the linear dependency as expected within the strong coupling regime. ${ }^{21,28}$

\section{B. Measurements of the ME occupation}

To study the scattering dynamics via ME occupation, the fluorescence data were measured as a function of the SPP incoupling angle $\varphi$. To separate the fluorescence from the luminescence of the polaritons, the D2 data were collected via an additional polarizer (see Fig. 1). The results from the sample NR2 are shown in Fig. 4(a) for several angles and for both TM and TE polarizations.

Since the SPPs as well as the polaritons always have a TM character, the measured TE signal is purely due to the ME fluorescence, as also clearly visible from the spectra of Figs. 4(a) and 4(c). As the molecules in the polymer film are randomly oriented, the fluorescence is unpolarized, i.e., the TE and TM spectra coincide, and the luminescence of the genuine polaritons can be obtained by subtracting the measured TE spectrum from the TM one, as shown in Fig. 4(b). This subtraction also clearly reveals the Rabi splitting at $\sim 570 \mathrm{~nm}$.

As a measure of the excited molecular population, we integrate the total fluorescence intensity of the TE spectra as a function of the coupling angle $\varphi$. The results are presented in Fig. 5(a), revealing a clear maximum around an angle of $55^{\circ}$ which is attributed to an efficient energy transfer to the MEs. We also show results of calculations based on the transfer matrix method. Here we use the Poynting vector to compute the energy transported into the NR film, where it becomes absorbed as MEs, which subsequently decay and are monitored in fluorescence. Overall, the measured and calculated results are in very good agreement, with minor deviations at the smaller angles probably due to measurement uncertainties caused by the small fluorescence intensities and a finite angle distribution of the excitation light, not taken into account in the theory.

\section{Three-level model}

To understand better the physics of the increased energy transfer around $\varphi \approx 55^{\circ}$, we next employ a generic quantum mechanical three-level model for the fluorescence. First, the measured dispersion curves are computed from the eigenmodes of the Hamiltonian

$$
H=\left(\begin{array}{ccc}
E_{\mathrm{SPP}}(k) & C_{1} & C_{2} \\
C_{1} & E_{1} & 0 \\
C_{2} & 0 & E_{2}
\end{array}\right),
$$

which is expressed in the basis of eigenstates for the SPP and the two molecular excitations. Here $E_{\mathrm{SPP}}(k)$ is the energy of 

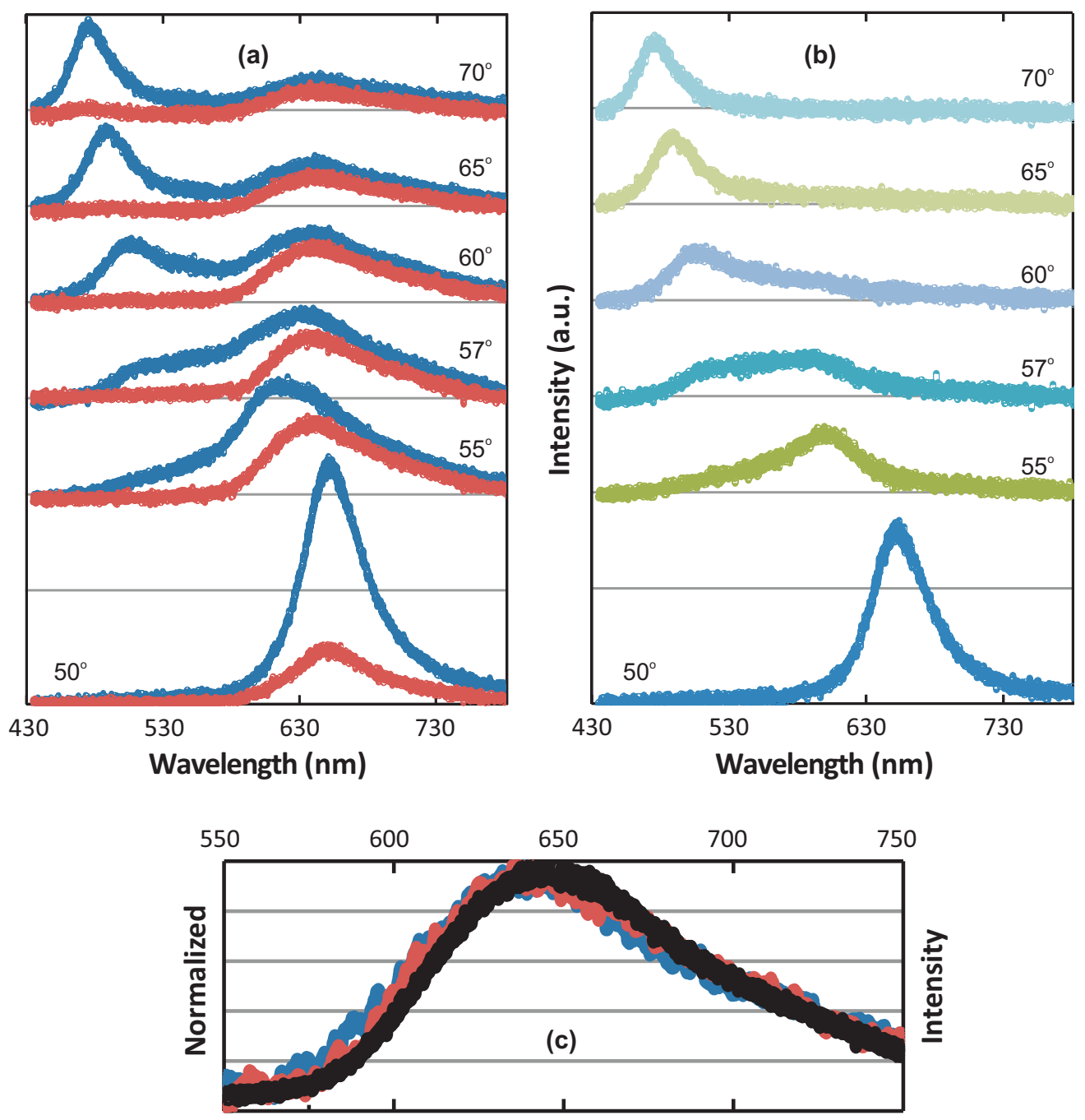

FIG. 4. (Color online) D2 spectra measured from the sample NR2 at different angles and polarizations. (a) Measured TE (red/light gray) and TM (blue/dark gray) D2 spectra at different angles. The TE spectra match well with the measured fluorescence spectrum of the NR [see (c)]. (b) Pure polariton luminescence obtained by subtraction of the spectra TM-TE. Note that the spectra with different angles are shifted vertically for clarity both in (a) and (b) (gray horizontal lines as zero levels). (c) Measured TE (red/light gray) and TM (blue/dark gray) D2 spectra for the excitation with a $488 \mathrm{~nm}$ laser from the silver side at the angle where the corresponding polaritons are launched, together with a measured fluorescence of a plain NR/SU-8 film (black).

the SPP for a given wave number $k$, i.e., SPP dispersion, $E_{1}$ and $E_{2}$ are the energies of the two MEs obtained from the absorption, and $C_{1}$ and $C_{2}$ are the coupling strengths between the SPP and the MEs.

From the Hamiltonian (1) one obtains through diagonalization the polariton eigenstates with $k$-dependent coefficients $\alpha$, $\beta$, and $\gamma$,

$$
\left|\Psi_{n}(k)\right\rangle=\alpha_{n}(k)|\mathrm{SPP}\rangle+\beta_{n}(k)\left|\mathrm{ME}_{1}\right\rangle+\gamma_{n}(k)\left|\mathrm{ME}_{2}\right\rangle,
$$

where $n$ labels the different eigenstates, i.e., LPB, MPB, and UPB. By fitting the coupling strengths $C_{i}$, a precise correspondence can be obtained between the eigenenergies $E_{n}$ and the energies of the measured polariton modes as shown in Fig. 6.

In the following, we assume that the excitation strength of each polariton branch is proportional to its plasmonic component, given by the coefficient $\alpha$, and ignore any leakage of light through the silver film, which is a fair assumption in the present setup. On the other hand, the total probability for molecular excitation is given by $\beta^{2}+\gamma^{2}$. These MEs will subsequently undergo vibrational relaxation and fluoresce at lower energies. Thus, the probability that the polariton mode $n$ becomes excited at a certain incoupling angle $\varphi$ and subsequently undergoes fluorescence through the ME is proportional to the product $\alpha_{n}^{2}\left(\beta_{n}^{2}+\gamma_{n}^{2}\right)$. The full fluorescence intensity is obtained by summing over all three branches. The corresponding results are shown in Fig. 5(b) displaying again good agreement with the experimental data.

\section{Analyzing mutual scatterings}

The simple three-level model allows us to also analyze the situation where the excited polariton population would thermalize prior to fluorescence. In this case, the branches are populated with a thermal distribution, according to the 

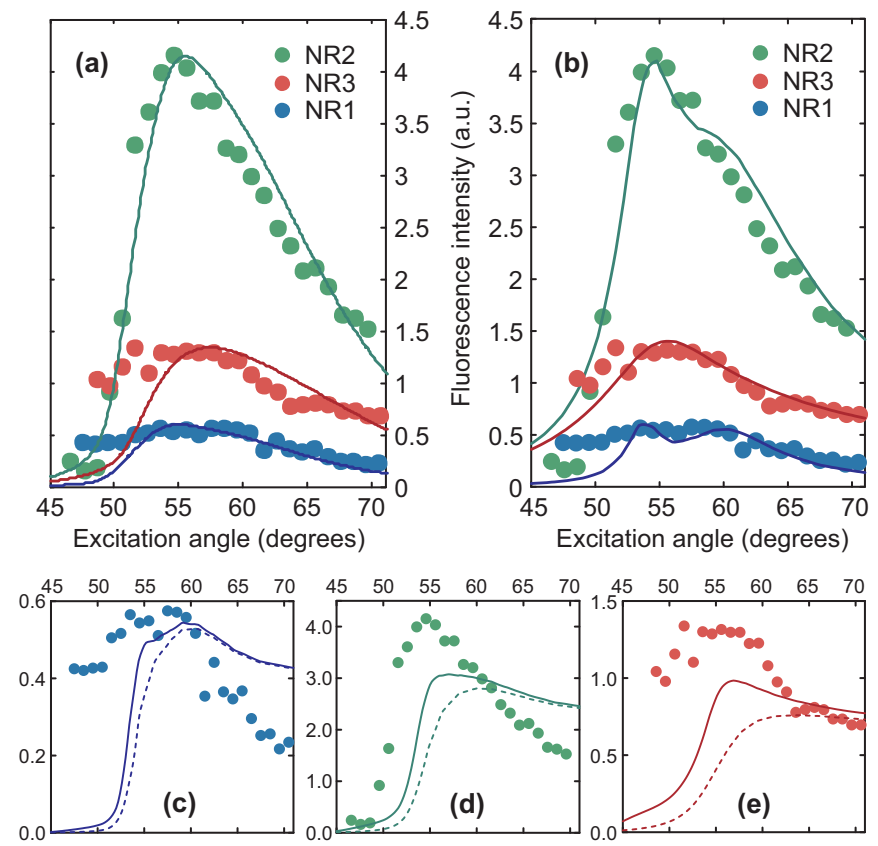

FIG. 5. (Color online) Fluorescence as a function of the incoupling angle. (a) Measured total fluorescence (circles) for the three samples NR1 (blue), NR2 (green), and NR3 (red), together with the theoretical transfer matrix calculations (solid lines). (b) Measured total fluorescence (circles) together with the results of the quantum mechanical three-state model (solid lines). (c)-(e) Measured total fluorescence (circles) of (c) NR1, (d) NR2, and (e) NR3 together with the results of the three-state model including relaxation and thermalization of the polariton modes (dashed lines), as well as decoherence via thermalization (solid lines).

excited energies $E_{n}\left(k_{n}\right)$ (see Fig. 6). We again assume that the $\mathrm{ME}$ component of the different polaritons is proportional to the fluorescence. For more details see the Appendix. Results including such thermalization are presented in Figs. 5(c)-5(e) (dashed lines) for all three samples. As one can clearly see, they do not agree at all with the measurements. This demonstrates that the scattering from the polariton states to MEs occurs much faster than thermalization of the polaritons.

Above we assumed that the ME occupation and thus the fluorescence is proportional to $\beta^{2}+\gamma^{2}$. This corresponds to the situation where the decoherence of the polaritons to a statistical distribution of its $\mathrm{ME}$ and SPP components happened via pure dephasing or internal molecular relaxation, without affecting the probability amplitudes $\alpha^{2}, \beta^{2}$, and $\gamma^{2}$. However, since the SPP and the MEs are strongly coupled to each other, one can a priori not rule out that decoherence also involves relaxation prior to fluorescence. To study such a possibility, we consider the situation where each polariton mode $n$ thermalizes and replace $\alpha_{n}, \beta_{n}$, and $\gamma_{n}$ by the corresponding thermal distributions, while still relating the excitation of the modes to the initial $\alpha_{n}$ value. The results for this model are also presented in Figs. 5(c)-5(e) (solid lines). As one can clearly see, they do not agree with the measurements either, proving that the scattering/decoherence from the polariton states to MEs happens faster than any thermalization.

To further study the time scale of internal molecular relaxation, we altered the experimental setup to a single wavelength SPP excitation by replacing the white light source with a $488 \mathrm{~nm}$ laser, and adjusted the angle so that only the corresponding polaritons are launched. The resulting D2 spectrum is shown in Fig. 4(c) together with a measured fluorescence spectrum for the NR film. As can be clearly seen, the D2 measurement exhibits pure fluorescence without any luminescence of polariton branches (the UPB could not be measured due to overlap with the laser). This demonstrates again that the UPB scatters only to the MEs and the scattering to other polariton branches is negligible. The result was obtained with several other molecules also (data not shown).

Similar phenomena have also been shown for polaritons of cavity photons strongly coupled to molecules, where the UPB decays with a time constant of several 100 fs to the ME. ${ }^{27}$ On the contrary, the scattering from the ME to LPB was shown to be much slower than the usual time scale of the vibrational relaxation. ${ }^{27}$ Our laser excitation result implies a similar dynamics for the SPP-ME polaritons. This would also
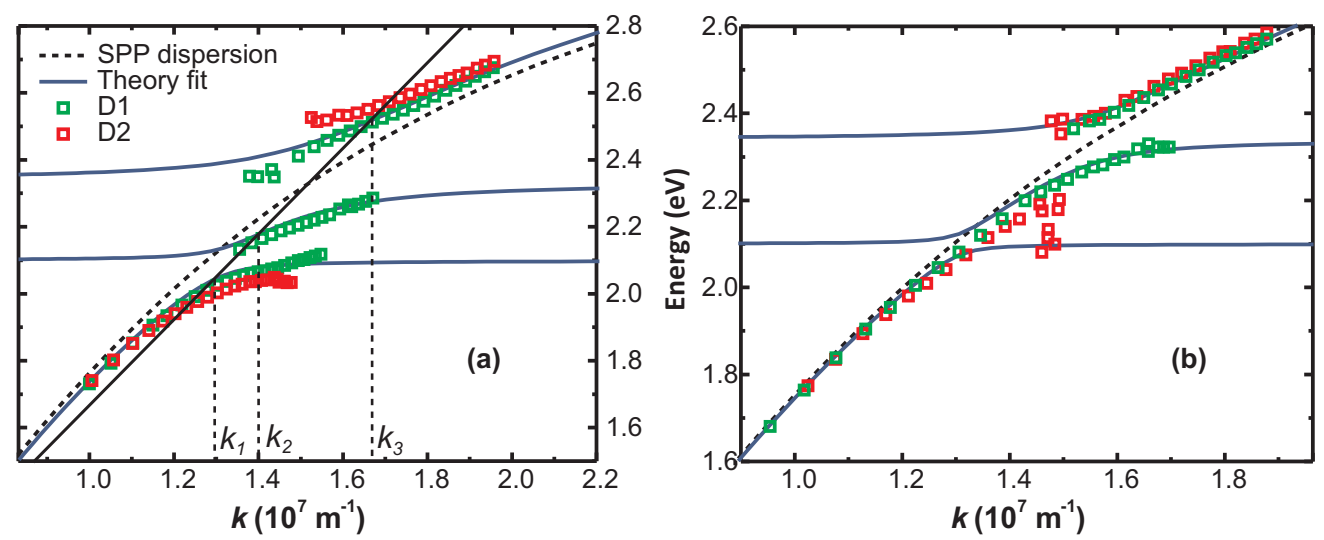

FIG. 6. (Color online) Dispersion curves for the samples (a) NR3 and (b) NR2, measured via reflectance D1 (green/light-gray squares) and luminescence D2 (red/dark gray squares). Solid blue lines are fits by the coupled oscillator model and the dotted black line is the calculated dispersion of SPP without NR. In (a) an example of the angle dependent light line of the excitation light within the prism is also shown as a solid black line. Launching of the polaritons happens on the marked $k_{n}$ values at the crossings of the dispersion and the light line. 
explain the observed decrease of the UPB luminescence in previous measurements. ${ }^{18-20}$

\section{CONCLUSIONS}

In conclusion, we have shown that the SPP-ME polaritons, i.e., SPPs strongly coupled to molecular excitations of Nile Red, solely decay through an internal molecular relaxation, and the process occurs in absence of any prior mutual polariton scattering or relaxation. In addition, single wavelength SPP studies further clarify that there is a negligible amount of energy transfer between the polariton branches, and the decrease of the UPB luminescence is due to fast decay to the MEs.

\section{ACKNOWLEDGMENTS}

This work was financed by Academy of Finland (Projects No. 135193 and No. 218182) and the Austrian Science Fund FWF under Project P24511-N26. Support from EU's COST action MP0803 is also greatly acknowledged.

\section{APPENDIX: THREE-LEVEL MODEL WITH THERMALIZATIONS}

To calculate the emission probability/intensity for each angle of excitation, the dispersion curves obtained from the D1 Kretchmann measurements were first fitted with the model of coupled oscillators or strong coupling between MEs and SPP. The Hamiltonian written in the basis formed of the SPP eigenstate $|\mathrm{SPP}\rangle$, and the two ME absorption bands $\left|\mathrm{ME}_{1}\right\rangle$ and $\left|\mathrm{ME}_{2}\right\rangle$, is shown in Eq. (1). Diagonalization of the Hamiltonian yields three eigenstates $\left|\Psi_{n}(k)\right\rangle$, and corresponding eigenenergies $E_{n}(k)$, as a function of wave vector $k(n=1,2,3)$, as illustrated in Fig. 6 and in Eq. (2). The states show clear avoided crossings, i.e., Rabi splittings at the crossings of the SPP dispersion and the $k$-independent MEs at $E_{1}=2.17 \mathrm{eV}$ and $E_{2}=2.38 \mathrm{eV}$, revealing forming of new ME-SPP-polariton states.

Three polariton branches were used for fitting in all the samples, even when the Rabi splitting at the low-energy ME could not be resolved in lower concentration samples. In this case only the coupling coefficient of the lower excitation was set to an estimated value below the experimental detection limit, yielding invisible small splitting as shown in Fig. 6. In other words, for each angle of excitation in the Kretschmann configuration three polariton eigenstates were always excited. The effect of the absolute value of this estimated small coupling, within reasonable limits, had a negligible effect to the result and only slightly affected the width of the maximum fluorescence in Fig. 5.

We assume that there is no leakage of direct light through the silver layer. Thus, the excitation strength/probability of each mode is proportional to its plasmonic component, described by the coefficient $\alpha$ since this is the only way energy can be transported through the silver film. Furthermore, after launching of the polariton it propagates and at some point either dissipates, scatters, or loses its coherence due to dephasing, thus becoming a statistical ensemble of the SPPs and the MEs. We assume here that the probability to scatter or dissipate is the same for all polaritons, independent of the wave vector or energy, and thus only affects the final emission probability by a constant factor.

No thermalization: Assuming that the decay of a polariton to a statistical distribution of its components $|\mathrm{SPP}\rangle,\left|\mathrm{ME}_{1}\right\rangle$, and $\left|\mathrm{ME}_{2}\right\rangle$ happens faster than any mutual scattering between them, the probability to end up in either of the MEs is $\beta^{2}+$ $\gamma^{2}$. These excitations will undergo vibrational relaxation and fluoresce at the lower energies, with a probability determined by the quantum efficiency of the molecule. Thus the probability for each polariton to be excited at a given excitation angle and fluoresce afterwards is related to the product $\alpha_{n}^{2}\left(\beta_{n}^{2}+\gamma_{n}^{2}\right)$. Taking into account all of the three launched polaritons, the full fluorescence probability for a given excitation angle $\varphi$ can be written as

$$
P(\varphi) \propto \sum_{n=1}^{3} \alpha_{n}^{2}\left(k_{n}\right)\left[\beta_{n}^{2}\left(k_{n}\right)+\gamma_{n}^{2}\left(k_{n}\right)\right],
$$

where the different $k_{n}$ values are indicated in Fig. 6. The calculated probabilities together with the measured fluorescence intensities are plotted in Fig. 5(b), and they agree well with each other.

Thermalization between the polaritons: To analyze the situation where the mutual scattering between the excited polaritons is so strong that they first thermalize and then undergo decoherence and fluoresce, we populate the polariton branches with a thermal distribution according to the excited energies $E_{n}(k)$, and again assume that the ME component of the different polaritons is proportional to the fluorescence intensity. The initial excitation strength for each polariton is again related to $\alpha^{2}$, so the total excitation strength for all the polaritons is proportional to the sum $\Lambda=\alpha_{1}^{2}\left(k_{1}\right)+\alpha_{2}^{2}\left(k_{2}\right)+$ $\alpha_{3}^{2}\left(k_{3}\right)$. Thus, the total fluorescence reads

$$
P(\varphi) \propto \Lambda \sum_{n=1}^{3} \frac{e^{-\frac{E_{n}}{k_{\mathrm{B}} T}}}{\sum_{i=1}^{3} e^{-\frac{E_{i}}{k_{\mathrm{B}} T}}}\left[\beta_{n}^{2}\left(k_{n}\right)+\gamma_{n}^{2}\left(k_{n}\right)\right] .
$$

Results for such thermalization are presented in Figs. 5(c)-5(e) for all three samples. The results do not agree at all with the measurements proving that scattering from the polaritons states to MEs occurs much faster than thermalization among them.

Thermalization under wave number conservation: In principle, decoherence can also involve internal relaxation and lead to thermalization between the components $|\mathrm{SPP}\rangle,\left|\mathrm{ME}_{1}\right\rangle$, and $\left|\mathrm{ME}_{2}\right\rangle$ of each polariton. Here the probability to be in ME and fluoresce is again $\left(\beta_{n}^{\prime 2}+\gamma_{n}^{\prime 2}\right)$, except now the coefficients $\beta_{n}^{\prime}$ and $\alpha_{n}^{\prime}$ are calculated from the thermal distribution between the energies $E_{\mathrm{SPP}}\left(k_{n}\right), E_{1}$, and $E_{2}$ of the polariton state. With $\alpha_{n}^{2}$ still being the excitation probability, the full fluorescence probability is again calculated by summing over all three polariton branches

$$
P(\varphi) \propto \sum_{n=1}^{3} \frac{\alpha_{n}^{2}\left(k_{n}\right)}{Z}\left[e^{-\frac{E_{1}}{k_{\mathrm{B}} T}}+e^{-\frac{E_{2}}{k_{\mathrm{B}} T}}\right],
$$

where $Z=e^{-E_{\mathrm{SPP}}\left(k_{n}\right) / k_{\mathrm{B}} T}+e^{-E_{1} / k_{\mathrm{B}} T}+e^{-E_{2} / k_{\mathrm{B}} T}$ is the partition function. The results are plotted in Figs. 5(c), 5(d), and 5(e) showing clear disagreement with the measurements. 
*j.jussi.toppari@jyu.fi

${ }^{1}$ D. K. Gramotnev and S. I. Bozhevolnyi, Nat. Photon. 4, 83 (2010).

${ }^{2}$ J. A. Schuller, E. S. Barnard, W. Cai, Y. C. Jun, J. S. White, and M. L. Brongersma, Nat. Mater. 9, 193 (2010).

${ }^{3}$ W. L. Barnes, A. Dereux, and T. W. Ebbesen, Nature (London) 424, 824 (2003).

${ }^{4}$ A. V. Zayats, I. I. Smolyaninov, and A. A. Maradudin, Phys. Rep. 408, 131 (2005).

${ }^{5}$ H. Raether, in Surface Plasmons, edited by G. Hohler (Springer, Berlin, 1988).

${ }^{6}$ W. L. Barnes, J. Mod. Opt. 45, 661 (1998).

${ }^{7}$ R. M. Amos and W. L. Barnes, Phys. Rev. B 59, 7708 (1999).

${ }^{8}$ J. R. Lakowicz, J. Malicka, I. Gryczynski, and Z. Gryczynski, Biochem. Biophys. Res. Commun. 307, 435 (2003).

${ }^{9}$ M. Albrecht and J. Creighton, J. Am. Chem. Soc. 99, 5215 (1977).

${ }^{10}$ H. Ditlbacher, A. Hohenau, D. Wagner, U. Kreibig, M. Rogers, F. Hofer, F. R. Aussenegg, and J. R. Krenn, Phys. Rev. Lett. 95, 257403 (2005).

${ }^{11}$ S. I. Bozhevolnyi, V. S. Volkov, E. Devaux, J.-Y. Laluet, and T. W. Ebbesen, Nature (London) 440, 508 (2006).

${ }^{12}$ H. Ditlbacher, J. R. Krenn, G. Schider, A. Leitner, and F. R. Aussenegg, Appl. Phys. Lett. 81, 1762 (2002).

${ }^{13}$ D. E. Chang, A. S. Srensen, E. A. Demler, and M. D. Lukin, Nat. Phys. 3, 807 (2007).

${ }^{14}$ R. A. Pala, K. T. Shimizu, N. A. Melosh, and M. L. Brongersma, Nano Lett. 8, 1506 (2008).

${ }^{15}$ D. Pacifici, H. J. Lezec, and H. A. Atwater, Nat. Photon. 1, 402 (2007).

${ }^{16}$ N. T. Fofang, N. K. Grady, Z. Fan, A. O. Govorov, and N. J. Halas, Nano Lett. 11, 1556 (2011).

${ }^{17}$ A. M. Glass, P. F. Liao, J. G. Bergman, and D. H. Olson, Opt. Lett. 5, 368 (1980).

${ }^{18}$ J. Bellessa, C. Bonnand, J. C. Plenet, and J. Mugnier, Phys. Rev. Lett. 93, 036404 (2004).

${ }^{19}$ T. K. Hakala, J. J. Toppari, A. Kuzyk, M. Pettersson, H. Tikkanen, H. Kunttu, and P. Törmä, Phys. Rev. Lett. 103, 053602 (2009).

${ }^{20}$ S. V. Baieva, T. K. Hakala, and J. J. Toppari, Nanoscale Res. Lett. 7, 191 (2012).

${ }^{21}$ J. Dintinger, S. Klein, F. Bustos, W. L. Barnes, and T. W. Ebbesen, Phys. Rev. B 71, 035424 (2005).
${ }^{22}$ F. Valmorra, M. Bröll, S. Schwaiger, N. Welzel, D. Heitmann, and S. Mendacha, Appl. Phys. Lett. 99, 051110 (2011).

${ }^{23}$ T. Schwartz, J. A. Hutchison, C. Genet, and T. W. Ebbesen, Phys. Rev. Lett. 106, 196405 (2011).

${ }^{24}$ A. Salomon, R. J. Gordon, Y. Prior, T. Seideman, and M. Sukharev, Phys. Rev. Lett. 109, 073002 (2012).

${ }^{25}$ A. Salomon, C. Genet, and T. W. Ebbesen, Angew. Chem. Int. Ed. 48, 8748 (2009).

${ }^{26}$ R. Houdré, R. P. Stanley, and M. Ilegems, Phys. Rev. A 53, 2711 (1996).

${ }^{27}$ T. Virgili, D. Coles, A. M. Adawi, C. Clark, P. Michetti, S. K. Rajendran, D. Brida, D. Polli, G. Cerullo, and D. G. Lidzey, Phys. Rev. B 83, 245309 (2011).

${ }^{28}$ D. G. Lidzey, A. M. Fox, M. D. Rahn, M. S. Skolnick, V. M. Agranovich, and S. Walker, Phys. Rev. B 65, 195312 (2002).

${ }^{29}$ G. H. Lodden and R. J. Holmes, Phys. Rev. B 83, 075301 (2011).

${ }^{30}$ E. Kretschmann, Z. Phys. 241, 313 (1971).

${ }^{31}$ G. P. Wiederrecht, G. A. Wurtz, and J. Hranisavljevic, Nano Lett. 4, 2121 (2004).

${ }^{32}$ S. Aberra Guebrou, C. Symonds, E. Homeyer, J. C. Plenet, Yu. N. Gartstein, V. M. Agranovich, and J. Bellessa, Phys. Rev. Lett. 108, 066401 (2012).

${ }^{33}$ G. P. Wiederrecht, J. E. Hall, and A. Bouhelier, Phys. Rev. Lett. 98, 083001 (2007)

${ }^{34}$ P. Vasa, R. Pomraenke, G. Cirmi, E. De Re, W. Wang, S. Schwieger, D. Leipold, E. Runge, G. Cerullo, and C. Lienau, ACS Nano 4, 7559 (2010).

${ }^{35}$ P. Vasa, W. Wang, R. Pomraenke, M. Lammers, M. Maiuri, C. Manzoni, G. Cerullo, and C. Lienau, Nat. Photon. 7, 128 (2013).

${ }^{36}$ T. Schwartz, J. A. Hutchison, J. Léonard, C. Genet, S. Haacke, and T. W. Ebbesen, Chem. Phys. Chem. 14, 125 (2013).

${ }^{37}$ A. Kuzyk, M. Pettersson, J. J. Toppari, T. K. Hakala, H. Tikkanen, H. Kunttu, and P. Törmä, Opt. Express 15, 9908 (2007).

${ }^{38}$ T. K. Hakala, J. J. Toppari, M. Pettersson, A. Kuzyk, H. Tikkanen, H. Kunttu, and P. Törmä, Appl. Phys. Lett. 93, 123307 (2008).

${ }^{39}$ A.-Y. Jee, S. Park, H. Kwon, and M. Lee, Chem. Phys. Lett. 477, 112 (2009).

${ }^{40}$ R. W. Christy and P. B. Johnson, Phys. Rev. B 6, 4370 (1972).

${ }^{41}$ V. Savona, L. C. Andreani, P. Schwendimann, and A. Quattropani, Solid State Commun. 93, 733 (1995). 